\section{Unravelling heterochromatin}

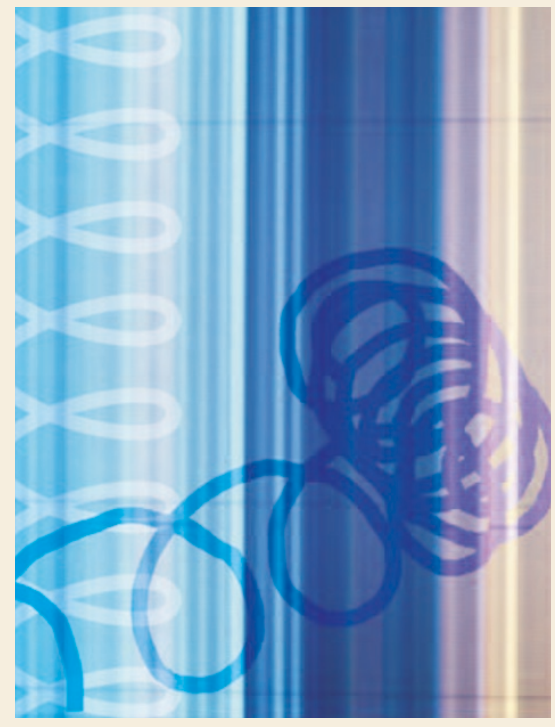

For a long time, heterochromatin was considered to be the 'dark matter' of the genome: highly condensed, late replicating and associated with lack of gene expression. Various lines of evidence indicated that heterochromatin could lead to transcriptional silencing, but the mechanism structure and function. chromatin structure.

\section{An array of opportunities}

Until the mid-1990s, studies of gene expression were limited to measuring transcription from one or a few genes. But then a tool arrived that changed all this, allowing the study of hundreds or thousands of transcripts at a time. This technology - the expression microarray - has revolutionized many areas of biology, from basic research to the understanding and treatment of human disease.

The study that brought microarrays to the attention of most researchers was published in 1995 by Patrick Brown and colleagues at Stanford University in California. They used an automated method to print 45 Arabidopsis thaliana cDNAs onto a glass slide. This 'array' was probed with a mixture of fluorescently labelled cDNAs that were derived from the reverse transcription of mRNAs extracted from a tissue sample. The amount of hybridization was unknown. Studies of position-effect variegation (PEV) in fruitflies, and of the centromeric and mating-type regions in fission yeast, have proved key to shedding light on the mysteries of heterochromatin

PEV - a clonally inherited pattern of active and silent gene states - was first described by Hermann Muller in 1930. Later, it was shown that affected silenced genes had been translocated to lie in close proximity to heterochromatin. In 1990, Sarah Elgin and colleagues identified heterochromatin protein 1 (HP1) and showed that mutations in the gene that encodes this protein in fruitflies behave as dominant suppressors of PEV, implicating HP1 in generating normal

Five years later, the same group showed that altered chromatin packaging had a role in PEV. They used a mobile transposable element - a popular tool among fruitfly geneticists - to insert a marker gene at various locations throughout the genome. PEV was seen for transgenes that inserted near centromeres, telomeres and on chromosome IV, which is heterochromatic in the fruitfly. Careful analysis of transgene

- and, therefore, the level of expression - was determined by measuring the intensity of fluorescence for each gene.

Although this was considered important in terms of the numbers of genes that could be studied, the fundamental implications for interpreting gene-expression patterns were not immediately appreciated. These became clear from later studies, such as a paper published in 1998 that used microarrays to monitor the expression of 800 genes throughout the yeast cell cycle. Subtle changes in overall geneexpression patterns over time were revealed that could not have been detected by other methods, even if dozens of genes had been studied.

By allowing different cell types to be accurately distinguished on the basis of expression patterns, microarrays have also had a

\footnotetext{
"The development of microarray technology has unequivocally revolutionized the way we examine and interpret gene expression."

James Broach
}

expression and chromatin structure showed that loss of expression was associated with an altered nucleosome array and reduced accessibility of restriction enzymes to the promoter. So, altered chromatin conformation underlies PEV, at least around the centromeres.

Studies of pericentromeric chromatin in fission yeast brought some important revelations about heterochromatin formation seven years later. The groups of Robert Martienssen and Shiv Grewal found that deleting genes that encode key components of the RNA interference (RNAi) machinery resulted in accumulation of complementary transcripts from centromeric heterochromatic repeats. Expression of centromeric transgenes in these deletion strains was de-repressed and histone $\mathrm{H} 3 \mathrm{~K} 9$ methylation - a mark of heterochromatin - was lost. These results raised the possibility that the RNAi machinery targets specific histone modifications to heterochromatic regions.

In a publication that followed two weeks later, the Grewal group turned to the mating-type region of fission yeast to study the factors that favour heterochromatin formation in cis. Little was known about them, except that repeat elements tended to be heterochromatic. This was an important issue - as was known from PEV, the heterochromatic state could spread to inactivate nearby genes. The authors found

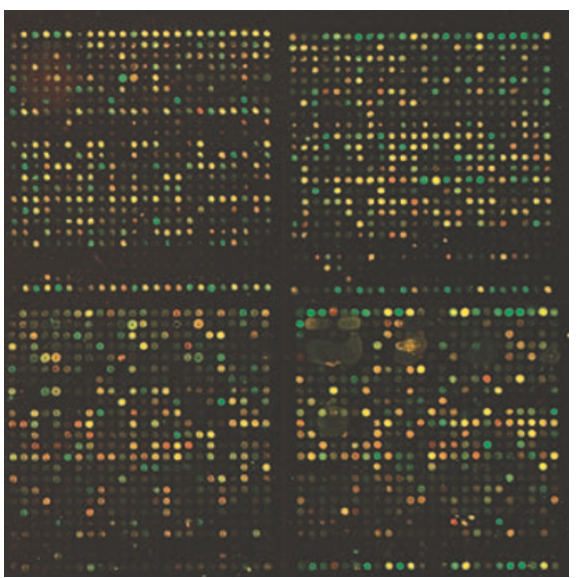

huge impact on medical research. In 2000, an impressive early example of this was reported, in which microarrays were used to study the expression of more than 8,000 genes in 65 human breast tumours. The authors generated 'molecular portraits' of gene expression that allowed them to distinguish between different classes of breast tumour and to identify two new categories that had been overlooked by traditional classification tools. They were also 
that $\operatorname{cen} H$, a centromere-homologous repeat that normally lies at the silent mating locus, is sufficient to bring about heterochromatin formation at ectopic sites. This ability is associated with $\mathrm{H} 3 \mathrm{~K} 9$ methylation and recruitment of Swi6 (a yeast HP1 counterpart) and, importantly, requires the RNAi machinery.

The dark matter of the genome was gradually becoming illuminated. The encouraging news was that the findings in fruitflies and fission yeast fitted with results from mammalian cells, in which we now know that the targeting of epigenetic modifications to repeats by the RNAi machinery also has a central role in heterochromatin formation and gene silencing.

Magdalena Skipper, Chief Editor, Nature Reviews Genetics

\section{References and links}

ORIGINAL RESEARCH PAPERS Wallarth, L. L. \&

Elgin, S. C. R. Position effect variegation in Drosophila is associated with an altered chromatin structure. Genes Dev. 9. 1263-1277 (1995) | Volpe, V. A. et al. Regulation of heterochromatic silencing and histone $\mathrm{H} 3$ lysine-9 methylation by RNAi. Science 297, 1833-1837 (2002) Hall, I. M. et al. Establishment and maintenance of a heterochromatin domain. Science 297, 2232-2237 (2002) FUTHER READING Muller, $\mathrm{H}$. J. Types of visible variations produced by X-rays in Drosophila melanogaster. J. Genet. 22, 299-234 (1930) | Eissenberg, J. C. et al. A mutation in heterochromatin-specific chromosomal protein associated with suppression of position effect variegation in Drosophila melanogaster. Proc. Natl Acad. Sci. USA 87, 9923-9927 (1990) | Matzke, M. A. \& Birchler, J. A. RNAi-mediated pathways in the nucleus. Nature Rev. Genet. 6, 24-35 (2005)

able to identify expression patterns that predicted the response to chemotherapy. In this way, microarrays have paved the way for new methods that allow the accurate classification and diagnosis of disease, and also indicate the most effective treatment strategies.

Since 1995, the microarray has swiftly changed from being regarded as a new, cuttingedge technology to being almost ubiquitous in biological research. The study of expression patterns across an entire genome has moved from an unattainable dream to an exciting reality that has fundamentally altered biology and medicine.

Louisa Flintoft, Associate Editor, Nature Reviews Genetics

References and links

ORIGINAL RESEARCH PAPERS Schena, M., Shalon, D. Davis, R. W. \& Brown, P. O. Quantitative monitoring of gene expression patterns with a complementary DNA microarray. Science 270, 467-470 (1995) | Spellman, P. T. et al. Comprehensive identification of cell cycle-regulated genes of the yeast Saccharomyces cerevisiae by microarray hybridization. Mol. Biol. Cell 9, 3273-3297 (1998) |

Perou, C. M. et al. Molecular portraits of human breast tumours. Nature 406, 747-752 (2000)

FURTHER READING van 't Veer, L. J. et al. Gene expression profiling predicts clinical outcome of breast cancer. Nature $\mathbf{4 1 5}$, 530-536 (2002)

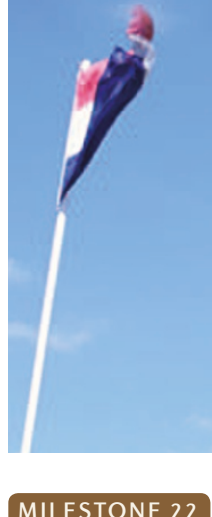

\section{Flagging histones}

Histones undergo a range of post-translational modifications, including acetylation, methylation, phosphorylation, ubiquitination and sumoylation. The idea that histone modifications, particularly acetylation and methylation, have fundamental roles in controlling gene transcription is now so firmly established that it is perhaps surprising to realize that this only became apparent during the past 5-10 years.

Histones had been known to be acetylated and methylated for a long time, and the idea of a function in transcription was considered as early as the 1960s. For example, in their 1964 paper, Allfrey, Faulkner and Mirsky demonstrated that histone acetylation can reduce their efficacy as inhibitors of transcription, and thought that this implied "a dynamic and reversible mechanism for activation as well as repression of RNA synthesis." In subsequent decades, enzymes that could acetylate histones were biochemically characterized and cloned. However, their relevance for regulating gene transcription was largely ignored.

In 1996, two papers - published within a month of each other - showed that histone acetylases and deacetylases were, in fact, wellknown transcriptional regulators. These studies provided the first clear connection between histone acetylation and transcriptional regulation. Both papers were hailed as breakthroughs and, from then on, understanding how histone modifications control chromatin structure and gene expression became a significant area of enquiry. In the first paper, Allis and colleagues set out to clone a histone acetyltransferase (HAT) from Tetrahymena thermophila. The cloned HAT turned out to have a high level of sequence relatedness to the yeast $\mathrm{Gcn} 5$, a transcriptional activator, with almost $80 \%$ sequence identity in some domains. Consistent with the sequence homology, the authors showed that recombinant Gcn5 had HAT activity in vitro. In the second study, Schreiber and colleagues purified mammalian histone deacetylases using an inhibitor
"The discovery of the first nuclear HAT is a landmark discovery which defines the modern era of chromatin research." Thomas Jenuwein

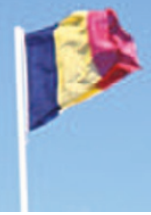

as an affinity matrix. This approach, combined with the microsequencing of purified proteins, yielded a surprise - one of the proteins had $60 \%$ sequence identity to yeast Rpd3, a characterized transcriptional repressor. The two antagonistic enzymatic activities had the opposite functional effects - activation or repression - on transcription.

So what about histone methylation? By 2000, several histone acetylases and deacetylases had been identified, but a functional link between histone methylation and chromatin structure or gene transcription remained elusive. Genetic screens in fruitflies and fission yeast had shown that the fruitfly suppressor of variegation 3-9 (Su(var)3-9) and fission yeast clr4 were important for establishing and propagating heterochromatin - a higher-order chromatin structure that is repressive for transcription. Work on the mammalian homologues had extended this link, but a mechanistic understanding of how these proteins controlled heterochromatin formation was lacking. In 2000, work from Jenuwein and colleagues showed that the mammalian homologue of $\mathrm{Su}$ (var)39 was a histone lysine methyltransferase that selectively methylated histone $\mathrm{H} 3$ at Lys9.

Together with earlier studies establishing a role for histone tails in transcriptional regulation (see Milestone 16), these three studies were pivotal for focusing the attention of the field on histone modifications and their importance as epigenetic markers.

Sowmya Swaminathan, Senior Editor, Nature Cell Biology

\section{References and links}

ORIGINAL RESEARCH PAPERS Brownell, J. E. et al. Tetrahymena histone acetyltransferase A: a homolog to yeast Gcn5p linking histone acetylation to gene activation. Cell 84, 843-851 (1996) | Taunton, J., Hassig, C. A. \& Schreiber, S. L. A mammalian histone deacetylase related to the yeast transcriptional regulator Rpd3p. Science 272, 408-411 (1996) Rea, S. et al. Regulation of chromatin structure by site-specific histone H3 methyltransferases. Nature 406, 593-599 (2000) FURTHER READING Allfrey, V., Faulkner, R. \& Mirsky, A Acetylation and methylation of histones and their possible role in the regulation of RNA synthesis. Proc. Natl Acad. Sci. USA 51 786-794 (1964) | Ruiz-Carillo, A., Wangh, L. J. \& Allfrey, V. G. Processing of newly synthesized histone molecules. Science 190, 117-128 (1975) | Kleff, S., Andrulis, E. D., Anderson, C. W. \& Sternglaz, R. Identification of a gene encoding a yeast histone H4 acetyltransferase. J. Biol. Chem. 270, 24674-24677 (1995) | Hebbes, T. R., Thorne, A. W. \& Crane-Robinson, C. A direct link between core histone acetylation and transcriptionally active chromatin. EMBO J. 5, 1395-1402 (1998) 